$$
\int_{\Gamma_{s}}|f(x+k)-f(x)|^{p} d V \leqslant C\|f\|_{m}^{p}|k|^{\nu}
$$

where $\gamma$ is a positive constant.

If the angle between $k$ and the tangent plane to $V_{s}$ is less than $\delta / 2$ then we can find another vector $k_{1}$ of length $\left|k_{1}\right|=\mid k_{\mid}$and such that the angles between $k-k_{1}$ and the tangent to $V_{s}+k$, and between $k_{1}$, and the tangent to $V_{s}$, are not less than $\delta / 2$ (if $V_{s}$ has the properties described in II then $V_{s}+k$ has these properties also provided that $k$ is short enough).

We get the vector $k_{1}$

$$
\begin{aligned}
& \left(\int_{\nabla_{s}}|f(x+k)-f(x)|^{p} d V\right)^{1 / p} \leqslant\left(\int_{V_{s}}\left|f(x+k)-f\left(x+k_{1}\right)\right|^{p} d V\right)^{1 / p} \\
& \leqslant\left(\int_{V_{s}}\left|f\left(x+k_{1}\right)-f(x)\right|^{p} d V\right)^{1 / p} \\
& =\left(\int_{\nabla_{s+k}}\left|f(x)-f\left(x+k-k_{1}\right)\right|^{p} d V\right)^{1 / p}+\left(\int_{V_{s}}\left|f(x)-f\left(x+k_{1}\right)\right|^{p} d V\right)^{1 / p}
\end{aligned}
$$

each of two above integrals can be estimated by means of Lemma 3 . Thus in both cases the integral under consideration tends uniformly to 0 and the proof is completed; the estimate of lemma 3 can obviously be extended by continuity to the whole of $W_{m}^{p}(\Omega)$.

\title{
References
}

[1] E. Ehrling, On a type of eigenvalue problems for certain elliptic differential operators, Mathematica Scandinavica 2 (1954), p. 267-287.

[2] K. K. Golovkin, DAN SSSR 134, No. 1 (1960), p. 19.22.

[3] L. Nirenberg, Remarks on strongly elliptic partial differential equations, Communications on Pure and Applied Mathematics 8 (1955), p. 649-675.

[4] S. L. Sobolev, Some applications of functional analysis in mathematical physics (in Russian), Moscov 1950.

[5] P. Szeptycki, A simple proof of an embedding theorem of the Kondrashev type, Bull. Acad. Pol. Sci., Série des Sci. math., astr. et phys., VI, No. 9 (1958), p. $561-564$

Reçu par la Rédaction le 14. 12. 1960

Approximation des opérateurs de J. Mikusiński par des fonctions continues

par

C. FOIAŞ (Bucureşti)

Soit $C[0, \infty)$ l'espace des fonctions continues définies sur $[0, \infty)$ muni de la topologie de la convergence uniforme sur tout compact de $[0, \infty)$. Par rapport à la convolution, $C[0, \infty)$ devient un domaine d'intégrité dont le corps $\mathfrak{X}$ des quotients formels $\frac{f}{g}, f, g \in C[0, \infty)$, est l'espace des opérateurs de J. Mikusiński ( $\left.{ }^{1}\right)$. Une suite $\left\{\frac{f_{n}}{g_{n}}\right\}$ converge vers $\frac{f}{g}$ (dans $\mathfrak{N}^{2}$ ), s'il existe des représentations $\frac{f_{n}}{g_{n}}=\frac{f_{n}^{\prime}}{g_{n}^{\prime}}, \frac{f}{g}=\frac{f^{\prime}}{g^{\prime}}$ telles que $f_{n}^{\prime} \rightarrow f^{\prime}($ dans $C[0, \infty))\left({ }^{2}\right)$.

Un problème naturel est de savoir si tout opérateur $\frac{f}{g} \epsilon \mathfrak{N}$ peut être approché par une suite $\left\{k_{n}\right\}, k_{n} \epsilon C[0, \infty)$. Il est évident que si $g$ est nulle dans un voisinage de l'origine, alors cela n'est pas toujours vrai. Dans cette note nous montrons que la propriété ci-dessus a lieu dès que $g$ n'est pas identiquement nulle au voisinage de l'origine.

Je tiens à remercier M. J. Mikusiński qui a bien voulu me proposer ce problème (au cours du Colloque d'Analyse Numérique tenu à Cluj, décembre 1960).

Nous commençons par établir une proposition préliminaire dont notre résultat sera une conséquence immédiate.

LEMME. Soient $f, g$ des fonotions sommables dans $[0, T], g$ non mulle presque partout au voisinage de 0 ; alors il existe une suite de fonctions $k_{n}$ continues dans $[0, T]$ telle que $\left\{g * t_{n}\right\}$ converge en moyenne vers $f$.

Démonstration. Dans le cas contraire, en vertu du théorème de Banach et de Hahn et du théorème sur la représentation des fonctionnelles linéaires continues sur l'espace des fonctions sommables sur $[0, T]$,

(1) Voir J. Mikusiński, Operational ealoulus 1959.

() Op. cit., p. 144 . 
il existerait une fonction $\Phi(t)$ mesurable et essentiellement bornée telle que

$$
\int_{0}^{T} f(t) \Phi(t) d t \neq 0
$$

$$
\int_{0}^{T}(g * k)(t) \Phi(t) d t=0
$$

\section{Weak* bases in conjugate Banach spaces}

by

I. SINGER (Bucharest)

quelle que soit la fonction continue $k$. En posant $\Psi(t)=\Phi\left(T_{T}^{\prime}-t\right)$, $\int_{0}^{T} k(t)(g * \Psi)(T-t) d t=k *(g * \Psi)(T)=((k * g) * \Psi)(T)=\int_{0}^{T}(g * k)(t) \Phi(t) d t=0$, d'ou l'on déduit que $g * \Psi=0$ presque partout. En vertu du théorème de Titchmarsh et de l'hypothèse faite sur $g$ on a $\Psi=0$ presque partout, ce qui contredit (1).

Nous pouvons maintenant démontrer notre

THÉORÈME. Pour tout opérateur $\frac{f}{g} \epsilon \mathfrak{N}$ tel que $g$ n'est pas identiquement nulle au voisinage de l'origine, il existe unè suite $\left\{k_{n}\right\}, k_{n} \in C[0, \infty)$, telle que $k_{n} \rightarrow \frac{f}{g}$ dans $\mathfrak{N}$.

Démonstration. D'après le Lemme, il existe des fonctions $k_{n}$ continues sur $[0, n]$ telles que

$$
\int_{0}^{n}\left|\left(g * k_{n}\right)(t)-f(t)\right| d t<\frac{1}{n} .
$$

Ceci entraîne $\left|1 * g * k_{n}-1 * f\right| \leqslant 1 / n$ pour $0 \leqslant t \leqslant n$. On peut supposer que $k_{n} \in C[0, \infty)$, ce qui ne restreint pas la généralité. Cela étant, on a $1 * g * \pi_{n l} \rightarrow 1 * f$ dans $C[0, \infty)$, donc

$$
k_{n}=\frac{1 * g * k_{n}}{1 * g} \rightarrow \frac{1 * f}{1 * g}=\frac{f}{g} \quad \text { dans } \quad \mathfrak{N},
$$

la division étant ici entendue comme l'opération inverse de la convolution.

Ajouté pendant la correction. Le résultat ci-dessus a été implicitement obtenu par M. I. Fenyő (qui nous a obligeamment attiré l'attention) dans son ouvrage $\hat{A}$ Mikusiński-féle operátorfogalom és disztribuició fogalma közti kapesolatról, Á Magyar Tud. Akad. Mat. és Fiz. Tud. Osztályának Kőzleményei 8 (1958), p. 385-392.

Reçu par la Rédaction le 24.12.1960 $\left\{x_{n}\right\}$ is called [2] a Schauder basis.
Introduction. The notion of a basis introduced by J. Schauder [8] has a natural extension to topological linear spaces [2]. A basis in a topological linear space $U$ is such a sequence $\left\{x_{n}\right\} \subset U$ that to every $x$ in $U$ there corresponds a unique sequence $\left\{a_{n}\right\}$ of scalars for which the following equation holds:

$$
x=\sum_{n=0}^{\infty} a_{n} x_{n} .
$$

Convergence of the series is that of the topology on $U$. Here the coefficients are obviously additive and homogeneous functionals on $U$ :

$$
a_{n}=\varphi_{n}(x), \quad n=1,2, \ldots
$$

When all these coefficient functionals are continuous on $U$, the basis

In the present paper we shall examine the particular case when $U$ is the conjugate space $E^{*}$ of a Banach space $E$ (over the real or complex field), endowed with its weak topology $\sigma\left(E^{*}, E\right)$; this space is locally convex. In this case we shall use for the bases and the Schauder bases of $U$, the terms : weak* basis and weak* Schauder basis of $E^{*}$, respectively. We shall also use the notation $\sum_{n=1}^{\infty}$ for the series in the weak topology $\sigma\left(E^{*}, E\right)$; thus,

$$
\sum_{n=1}^{\infty} g_{n}=g \quad\left(g_{n}, g \in E^{*}\right)
$$

means that

$$
\sum_{n=1}^{\infty} g_{n}(x)=g(x) \quad \text { for all } \quad x \in E
$$

In $\S 1$ we show an example of a weak* basis which is not a weak* Schauder basis. $\S 2$ contains our main result which is the construction of a $\sigma\left(E^{*}, E\right)$-separable conjugate Banach space $E^{*}$ in which there exists 